participate in a set of three interviews across the academic year. Thirty-two students (16 drinkers, 16 abstainers) were randomly selected to take part. 28 interviews were completed in the two weeks following FW, 26 at the end of the first semester and 20 at the end of the second semester. Equal numbers of drinkers and abstainers were interviewed at each stage. Interviews were digitally audio-recorded, transcribed verbatim and analysed using the Framework approach.

Results Most drinkers came to university with the view that getting drunk was acceptable; some already had an established pattern of regular binge drinking. Upon arrival at university drinking became a more frequent feature for all, for some occurring on a near-daily basis during FW. Most students reported drinking in higher quantities. This in part appeared to be driven by students' expectations that university socialising is centralised around drinking, as well as the provision of daily nightclub events by the Students' Union which received more promotion over non-drinking focused social opportunities prior to and during FW. Alcohol remained a frequent part of socialising after FW, with a continued emphasis on drinking opportunities over alternative activities. Several abstainers reported feeling socially excluded due to the dearth of nondrinking focused social opportunities across the year, although some were able to quickly form friendships with other nondrinkers. Both drinkers and abstainers highlighted a need for there to be less emphasis on drinking throughout the year.

Conclusion Although many students come to university with established drinking patterns and expectations, the environment fosters binge drinking at the start of and throughout the academic year. Although this study was based on one university, the findings corroborate those found at others. They will be used to create recommendations on how to reduce problematic drinking in this population.

\section{OP75 A QUALITATIVE EXPLORATION OF THE INTERSECTION BETWEEN SOCIAL INFLUENCE AND CULTURAL NORMS IN RELATION TO THE DEVELOPMENT OF ALCOHOL CONSUMPTION PRACTICES DURING ADOLESCENCE}

GJ MacArthur*, M Hickman, R Campbell. Department of Population Health Sciences, University of Bristol, Bristol, UK

\subsection{6/jech-2018-SSMabstracts.74}

Background Despite downward trends in alcohol consumption among young people in the UK, a substantial proportion drink and by the age of 17 , at least half of young people report weekly drinking. Evidence regarding effects of school-based interventions to prevent harmful alcohol use is mixed and there remains a need for qualitative research to inform intervention development. We sought to explore young people's perspectives on behavioural and cultural influences relating to alcohol use and the socio-cultural context around alcohol consumption during mid-adolescence.

Methods Forty-two young people ( $n=21$ males, $n=21$ females) were recruited from schools $(n=30$, aged $14-15$ years) and youth groups ( $n=12$, aged $14-18$ years) in the West of England. In schools, participants were randomly selected from year 10 (aged 14-15) with snowball sampling used to maximise diversity of alcohol use, while additional participants were recruited from youth groups via youth workers. Data were collected via semi-structured one-to-one $(n=25)$ and paired $(n=4)$ interviews and one focus group. Interviews were audio-recorded, transcribed verbatim and analysed thematically using NVivo 10.

Results Alcohol use was perceived as a normalised social practice in the wider population and parental influence, attitudes and the provision of alcohol underpinned adolescent attitudes and behaviour. In adolescence, alcohol consumption was associated with being cool, mature and popular, while enabling escape from reality and boosting confidence and enjoyment. Such positive expectancies alongside opportunity contributed to motivating initiation, but social influences were paramount for most, with participants describing a need to fit in or conform with friends to avoid social exclusion or derision. Such influences positioned drinking and intoxication at parties as a normative social practice, further providing opportunities for social learning and incentivising drinking through competition, associations with popularity, and a desire to avoid responsibility for intoxicated friends. Social media weaved into young people's lives the display of positive alcohol-associated depictions of social status, enjoyment and maturity. This intersection of influences, norms and incentives generated a pressurised environment, characterised by conformity being experienced as an obligation to drink, and a sense of unease around abstinence which elicited stigmatising insults.

Conclusion Social influences, cultural norms, incentives and social media contribute to development of a pressurised environment around alcohol consumption during mid-adolescence, driving the escalation of alcohol use as a normative social practice. Our findings highlight the need to acknowledge normative influence and the drivers of cultural norms and practices when developing new interventions to prevent harmful alcohol use during adolescence.

\section{OP76 INVESTIGATING THE GROWING TREND OF NON- DRINKING AMONG YOUNG PEOPLE; ANALYSIS OF REPEATED CROSS-SECTIONAL SURVEYS IN ENGLAND 2005-2015}

L Ng Fat, N Shelton, N Cable*. Epidemiology and Public Health, University College London, London, UK

\subsection{6/jech-2018-SSMabstracts.75}

Background Non-drinking among young people has increased over the past decade in England, yet the underlying factors driving this change is unknown. Traditionally non-drinking has been found to be associated with lower socio-economic status and poorer health. This study explores among which subgroups non-drinking has increased, and how this correlates with changes in drinking patterns, to identify whether behaviours are becoming more polarised, or reduction is widespread among young people.

Methods Using repeated annual cross-sectional data on young people aged 16 to 24 years from the nationally representative Health Survey for England 2005-2015, trends in non-drinking including lifetime abstention and not drinking in the past week were explored using STATA15; 1) Trends in non-drinking among social-demographic and health sub-groups were examined through a test for linear time-trends among subgroups, adjusting for age. Additionally, an interaction between year and each variable was modelled in sex- and age-adjusted logistic regression models 2) Spearman correlation co-efficients were calculated between the proportion non-drinking by year, and heavy episodic drinking, and the mean alcohol units 
consumed on the heaviest drinking day. In addition, ordinary least squares regression analyses were used, modelling the proportion non-drinking as the independent variable, and the proportion binge/mean units as the dependent variable.

Results Rates of non-drinking increased from 18\% [95\% CI $16 \%$ to $22 \%$ ] in 2005 to $29 \%$ [25-33\%] in 2015 (test for trend; $\mathrm{p}<0.001)$, largely attributable to increases in lifetime abstention. Not drinking in the past week increased from $35 \%$ [32-39\%] to 50\% [45-55\%] $(\mathrm{p}<0.001)$. Significant linear increases in non-drinking were found among most subgroups including healthier sub-groups (non-smokers, those with high physical activity and good mental health), north and south regions, in full-time education, and employed. Among white participants, non-drinking increased from 14\% [12$17 \%$ ] in 2005 to $20 \%$ [17-24\%] in 2015. No significant increases in non-drinking were found among smokers, ethnic minorities and those with poor mental health. At the population-level, a one percentage point increase in non-drinking, predicted a 0.22 reduction in mean alcohol units consumed [95\% CI -0.32 to 0.12 ], and a 1.06 percentage point decrease in the proportion binge drinking [95\% CI 1.56 to 0.54]

Conclusion Increases in non-drinking were found with variables less commonly associated with non-drinking, suggesting this behaviour may be becoming more normative. This trend is to be welcomed from a public health standpoint and should be capitalised on going forward. Drinking and smoking continue to cluster and could be targeted in tandem. Future research should explore attitudes towards alcohol among young people.

\section{OP77 CAN MENTAL HEALTH COMPETENCE BUFFER AGAINST THE HIGHER RISK OF SMOKING INITIATION AMONG TEENAGERS WITH PARENTS WHO SMOKE? FINDINGS FROM THE UK MILLENNIUM COHORT STUDY}

${ }^{1,2} \mathrm{~A}$ Pearce* ${ }^{2} \mathrm{E}$ Rougeaux, ${ }^{3} \mathrm{~J}$ Deighton, ${ }^{2} \mathrm{RM}$ Viner, ${ }^{2} \mathrm{C}$ Law, ${ }^{2} \mathrm{~S}$ Hope. ${ }^{1} \mathrm{MRC} / \mathrm{CSO}$ Social and Public Health Sciences Unit, University of Glasgow, Glasgow, UK; ${ }^{2}$ Population, Policy and Practice Programme, UCL Great Ormond Street Institute of Child Health, London, UK; ${ }^{3}$ Evidence Based Practice Unit, UCL and the Anna Freud Centre, London, UK

\subsection{6/jech-2018-SSMabstracts.76}

Background Most smokers initiate before the age of eighteen, and smoking in adolescence is strongly influenced by parental smoking habits. Despite this, few studies have examined factors which may protect teenagers against the risk of having a smoking parent. We investigated whether skills-based components of positive mental health in childhood (Mental Health Competence, MHC) modified the association between parent and teenager smoking, using contemporary data from the UKrepresentative Millennium Cohort Study ( $\sim 18000$ children, born 2000-2002; analytic sample: $n=10,133)$.

Methods Cohort members (CMs) reported whether they had ever smoked cigarettes or e-cigarettes at 14 years(y). A dichotomised variable indicated whether one or both parents reported their own tobacco use when CMs were 11 y. A four-class latent measure of MHC captured learning skills and prosocial behaviours at $11 \mathrm{y}$ : High, High-Moderate, Moderate, Low.

We examined effect modification in two ways. First, we compared risk differences (RD; estimated using binary regression) for $\mathrm{CM}$ smoking according to parental smoking, across levels of MHC. Second, we estimated RDs for CM smoking according to combinations of parental smoking and MHC. Confounding by socio-economic and demographic characteristics and parent's mental health was adjusted for. Survey weights accounted for sample design and attrition; multiple imputation addressed item missingness.

Results Similar proportions of CMs had ever smoked cigarettes $(17 \%)$ and e-cigarettes (18\%), although overlap was moderate (40\% who had smoked either had smoked both). CMs were more likely to have ever smoked cigarettes if at least one parent smoked (RD: 16\%[13-184]) (baseline: no parents who smoke[11\%]). This elevated risk was observed across all levels of MHC, but was greatest for Low MHC (RD: 21\%[11-31] (RDs in other MHC groups: 7\%-12\%). When combining parental smoking and MHC (baseline: no smoking parent, high MHC), those with Low MHC and a smoking parent had a RD of $28 \%(20-36)$. This was higher than the sum of RDs in CMs with Low MHC but a non-smoking parent (7\%[114]) and with High MHC but a smoking parent (11\% [715]). Thus, Low MHC carried an excess risk. There was little evidence of effect modification by Moderate or High-Moderate MHC. Results were similar for e-cigarettes.

Conclusion The association between parent smoking and teenage smoking initiation was considerably stronger in those with Low MHC in contemporary, UK-representative data. These results require replication in other populations, and at older ages (examining progression to regular smoking), but imply that MHC improvement before transition to secondary school holds potential to buffer against an important smoking risk factor.

The views expressed are those of the authors and not necessarily those of the funders.

\section{Physical activity}

\section{OP78 ARE QUALITY OF LIFE AND FAMILY EXPENDITURE ON PHYSICAL ACTIVITY ASSOCIATED WITH PHYSICAL ACTIVITY IN 2-4-YEAR-OLD CHILDREN?}

${ }^{1} \mathrm{~L}$ Tinner*, ${ }^{1} \mathrm{R}$ Kipping, ${ }^{2} \mathrm{~J}$ White, ${ }^{3} \mathrm{R}$ Jago, ${ }^{1} \mathrm{C}$ Metcalfe, ${ }^{1} \mathrm{~W}$ Hollingworth. ${ }^{1}$ Population Health Sciences, University of Bristol, Bristol, UK; ${ }^{2}$ Centre for Trials Research, University of Cardiff, UK; ${ }^{3}$ School for Policy Studies, University of Bristol, UK

\subsection{6/jech-2018-SSMabstracts.77}

Background Despite the well-described health benefits associated with physical activity, many children do not engage in the recommended level of physical activity. To inform public health interventions, there is a need to determine factors associated with physical activity in children. We examined the extent to which the Pediatric Quality of Life Inventory (PedsQL) and family expenditure on physical activity were associated with minutes spent physically active and in moderate-to-vigorous physical activity (MVPA) per day in young children.

Methods Cross-sectional study with a sample of 81 children aged 2-4 years in the South West of England, taking part in the Nutrition and Physical Activity Self-Assessment for Child Care (NAPSACC UK) feasibility randomized controlled trial. We ran descriptive statistics, along with Student t-tests to determine differences by gender, age and parental education and compare physical activity on nursery and non-nursery days. The associations between physical activity, PedsQL scores (physical and psychosocial) and family expenditure on physical 